\title{
Large deviations for randomly weighted sums with dominantly varying tails and widely orthant dependent structure
}

\author{
Xiaodong Bai ${ }^{1 *}$, Lixing Song ${ }^{2}$ and Tao $\mathrm{Hu}^{3}$
}

*Correspondence:

baixd518@gmail.com

1 School of Science, Dalian

Nationalities University, Liaohe West

Road, Dalian, 116600, China

Full list of author information is

available at the end of the article

\begin{abstract}
We prove large deviation inequalities for the randomly weighted partial and random sums $S_{n}^{\theta}=\sum_{i=1}^{n} \theta_{i} X_{i}, n \geq 1 ; S_{c}^{\theta}(t)=\sum_{i=1}^{N(t)}\left(\theta_{i} X_{i}+c\right), c \in R$, where $\{N(t), t \geq 0\}$ is a counting process, $\left\{\theta_{i}, i \geq 1\right\}$ is a sequence of positive random variables with two-sided bounds, and $\left\{X_{i}, i \geq 1\right\}$ is a sequence of non-identically distributed real-valued random variables, while the three random sources above are mutually independent. Special attention is paid to the distribution of dominated variation and the widely orthant dependence structure.

MSC: Primary 62E20; secondary 62H20; 62P05
\end{abstract}

Keywords: large deviations; randomly weighted sums; widely orthant dependence; dominated variation

\section{Introduction}

Let $\left\{X_{k}, k \geq 1\right\}$ be a sequence of real-valued random variables (r.v.s) with $X_{k}$ 's distribution function (d.f.) $F_{k}(x)=1-\bar{F}_{k}(x)$ and $\mu_{k}=E X_{k}=0$ for every $k \geq 1$, and $\left\{\theta_{k}, k \geq 1\right\}$ be another sequence of positive random variables, satisfying $P_{r}\left(a \leq \theta_{k} \leq b\right)=1, k \geq 1$, where $0<a \leq b<\infty$. $\{N(t), t \geq 0\}$ denotes a counting process (that is, a non-negative, non-decreasing, and integer-valued stochastic process) with a finite mean function $\lambda(t)$ for $t \geq 0$ and $\lambda(t) \rightarrow \infty$ as $t \rightarrow \infty$. Besides, the three random sources above are mutually independent. Denote $S_{n}^{\theta}=\sum_{i=1}^{n} \theta_{i} X_{i}, n \geq 1$ and $S_{c}^{\theta}(t)=\sum_{i=1}^{N(t)}\left(\theta_{i} X_{i}+c\right), t \geq 0, c \in R$. By convention, the summation over an empty set of indices produces a value of 0 . In the present paper, we are interested in the probabilities of large deviations of $\left\{S_{n}^{\theta}\right\}$ and $\left\{S_{c}^{\theta}(t)\right\}$ in the situation that $\left\{X_{k}, k \geq 1\right\}$ are heavy-tailed and widely orthant dependent.

Since the theory of large deviations with heavy tails is widely used in insurance and finance, in recent decades, there have been a series of articles devoted to related problems. For more details, please refer to Embrechts et al. [1], Klüppelberg and Mikosch [2], Mikosch and Nagaev [3] and references therein. Recently Tang [4] extended the asymptotic behavior of large deviation probabilities of partial sums of heavy-tailed random variables to the case of negatively dependent ones. Under the assumption that random variables are non-identically distributed and extended negatively dependent, Liu [5] obtained a result similar to the one in the above paper, which was promoted to random sums in various situations later by Chen et al. [6]. Specially, Shen and Lin [7] investigated large

(O2014 Bai et al.: licensee Springer. This is an Open Access article distributed under the terms of the Creative Commons Attribution License (http://creativecommons.org/licenses/by/2.0), which permits unrestricted use, distribution, and reproduction in any medium, provided the original work is properly cited. 
deviations of randomly weighted partial sums with negatively dependent and consistently varying-tailed random variables, but, unfortunately, there are some flaws in their proofs.

In this paper, motivated by the work of Liu and $\mathrm{Hu}$ [8] and Chen et al. [6], on the one hand, we aim to prove that for each fixed $\gamma>0$, there exist positive constant $M_{1}$ and $M_{2}$ such that the inequalities

$$
P_{r}\left(S_{n}^{\theta}>x\right) \geq M_{1}(1+o(1)) \sum_{k=1}^{n} P_{r}\left(\theta_{k} X_{k}>x\right)
$$

and

$$
P_{r}\left(S_{n}^{\theta}>x\right) \leq M_{2}(1+o(1)) \sum_{k=1}^{n} P_{r}\left(\theta_{k} X_{k}>x\right)
$$

hold uniformly for all $x \geq \gamma n$ as $n \rightarrow \infty$, respectively; on the other hand, for arbitrarily fixed $\gamma>c$ ( $c$ is an arbitrarily given real number), there are positive constant $\tilde{M}_{1}$ and $\tilde{M}_{2}$ such that

$$
P_{r}\left(S_{c}^{\theta}(t)>x\right) \geq \tilde{M}_{1}(1+o(1)) E\left[\sum_{k=1}^{N(t)} P_{r}\left(\theta_{k} X_{k}>x-c \lambda(t)\right)\right]
$$

and

$$
P_{r}\left(S_{c}^{\theta}(t)>x\right) \leq \tilde{M}_{2}(1+o(1)) E\left[\sum_{k=1}^{N(t)} P_{r}\left(\theta_{k} X_{k}>x-c \lambda(t)\right)\right]
$$

hold uniformly for all $x \geq \gamma \lambda(t)$ as $t \rightarrow \infty$, respectively.

The paper is organized as follows. Section 2 presents our main results after recalling some preliminaries. Sections 3 and 4 prove Theorems 2.1 and 2.2, respectively.

\section{Main results}

We say that a random variable $X$ or its distribution function is heavy-tailed if $E e^{t X}=\infty$ for all $t>0$. An important class of heavy-tailed distributions is $\mathcal{D}$, which consists of all distributions with dominated variation in the sense that the relation $\lim _{\sup } \bar{x} \bar{F}(x y) / \bar{F}(x)<\infty$ holds for some (hence for all) $0<y<1$. Recall the upper/lower Matuszewska index of distribution $F$, defined as $J_{F}^{+}=-\lim _{y \rightarrow \infty}\left(\log \bar{F}_{*}(y) / \log y\right)$ and $J_{F}^{-}=-\lim _{y \rightarrow \infty}\left(\log \bar{F}^{*}(y) / \log y\right)$, where $\bar{F}_{*}(y)=\liminf _{x \rightarrow \infty} \bar{F}(x y) / \bar{F}(x)$ and $\bar{F}^{*}(y)=\limsup _{x \rightarrow \infty} \bar{F}(x y) / \bar{F}(x)$ for any $y>0$. From Lemma 3.5 of Tang and Tsitsiashvili [9], we know that if $F \in \mathcal{D}$, then $0 \leq J_{F}^{-} \leq J_{F}^{+}<\infty$, and for arbitrary $p_{1}<J_{F}^{-}$and $p_{2}>J_{F}^{+}$, there exist positive constant $\widetilde{C}_{i}$ and $\widetilde{D}_{i}, i=1,2$, such that

$$
\bar{F}(y) / \bar{F}(x) \geq \widetilde{C}_{1}(x / y)^{p_{1}}
$$

holds for all $x \geq y \geq \widetilde{D}_{1}$, and

$$
\bar{F}(y) / \bar{F}(x) \leq \widetilde{C}_{2}(x / y)^{p_{2}}
$$


holds for all $x \geq y \geq \widetilde{D}_{2}$. Hence, for any $p_{1}<J_{F}^{-}$, we have

$$
\bar{F}(x)=o\left(x^{-p_{1}}\right)
$$

and for any $p_{2}>J_{F}^{+}$,

$$
x^{-p_{2}}=o(\bar{F}(x)) .
$$

Furthermore, if the distribution $F^{+}(x)=F(x) \mathbf{1}_{(x \geq 0)}$ has a finite mean, then $J_{F}^{+} \geq 1$.

Now, we present some new dependence structures introduced in Wang et al. [10].

Definition 2.1 We say that the r.v.s $\left\{\eta_{n}, n \geq 1\right\}$ are widely upper orthant dependent (WUOD) if there exists a finite real sequence $\left\{g_{U}(n), n \geq 1\right\}$ satisfying for each $n \geq 1$ and for all $x_{i} \in(-\infty,+\infty), 1 \leq i \leq n$,

$$
P_{r}\left(\bigcap_{i=1}^{n}\left\{\eta_{i}>x_{i}\right\}\right) \leq g_{U}(n) \prod_{i=1}^{n} P_{r}\left(\eta_{i}>x_{i}\right) ;
$$

we say that the r.v.s $\left\{\eta_{n}, n \geq 1\right\}$ are widely lower orthant dependent (WLOD) if there exists a finite real sequence $\left\{g_{L}(n), n \geq 1\right\}$ satisfying for each $n \geq 1$ and for all $x_{i} \in(-\infty,+\infty)$, $1 \leq i \leq n$,

$$
P_{r}\left(\bigcap_{i=1}^{n}\left\{\eta_{i} \leq x_{i}\right\}\right) \leq g_{L}(n) \prod_{i=1}^{n} P_{r}\left(\eta_{i} \leq x_{i}\right) ;
$$

if they are both WUOD and WLOD, then we say that the r.v.s $\left\{\eta_{n}, n \geq 1\right\}$ are widely orthant dependent (WOD). WUOD, WLOD, and WOD r.v.s are called, by a joint name, wide dependence (WD) r.v.s, and $g_{U}(n), g_{L}(n), n \geq 1$, are called dominating coefficients.

Wang et al. [10] also gave some examples of WD r.v.s with various dominating coefficients which show that WD r.v.s contain some common negatively dependent r.v.s, some positively dependent r.v.s and some others.

From the definitions of WD, the following proposition can be obtained directly (see, e.g., Wang et al. [10]).

Proposition 2.1 (1) Let $\left\{\eta_{n}, n \geq 1\right\}$ be WUOD (WLOD) with dominating coefficients $g_{U}(n)$, $n \geq 1\left(g_{L}(n), n \geq 1\right)$. If $\left\{f_{n}(\cdot), n \geq 1\right\}$ are non-decreasing, then $\left\{f_{n}\left(\eta_{n}\right), n \geq 1\right\}$ are still WUOD (WLOD) with dominating coefficients $g_{U}(n), n \geq 1\left(g_{L}(n), n \geq 1\right)$; if $\left\{f_{n}(\cdot), n \geq 1\right\}$ are nonincreasing, then $\left\{f_{n}\left(\eta_{n}\right), n \geq 1\right\}$ are WLOD (WUOD) with dominating coefficients $g_{U}(n)$, $n \geq 1\left(g_{L}(n), n \geq 1\right)$.

(2) If $\left\{\eta_{n}, n \geq 1\right\}$ are non-negative and WUOD with dominating coefficients $g_{U}(n), n \geq 1$, then for each $n \geq 1$,

$$
E \prod_{i=1}^{n} \eta_{i} \leq g_{U}(n) \prod_{i=1}^{n} E \eta_{i} .
$$


In particular, if $\left\{\eta_{n}, n \geq 1\right\}$ are WUOD with dominating coefficients $g_{U}(n), n \geq 1$, then for each $n \geq 1$ and any $s>0$,

$$
E \exp \left\{s \sum_{i=1}^{n} \eta_{i}\right\} \leq g_{U}(n) \prod_{i=1}^{n} E \exp \left\{s \eta_{i}\right\} .
$$

For convenience, we introduce some notation. For two positive functions $a(\cdot)$ and $b(\cdot)$, we write $a(t) \asymp b(t)$ if $0<\liminf _{t \rightarrow \infty} a(t) / b(t) \leq \limsup _{t \rightarrow \infty} a(t) / b(t)<\infty$. For two positive bivariate functions $a(\cdot, \cdot)$ and $b(\cdot, \cdot)$, we say that the asymptotic relation $a(n, x) \lesssim b(n, x)$ or $a(n, x) \gtrsim b(n, x)$ holds uniformly over all $x$ in a nonempty set $\Delta$, if $\lim \sup _{n \rightarrow \infty} \sup _{x \in \Delta} a(n, x) / b(n, x) \leq 1$ or $\liminf _{n \rightarrow \infty} \inf _{x \in \Delta} a(n, x) / b(n, x) \geq 1$. For a real number $x$, we write $x^{+}=\max \{x, 0\}$ and $x^{-}=-\min \{x, 0\}$.

Before we state our main results, we will introduce some basic assumptions, to be used in this paper.

$\left(\mathrm{A}_{1}\right)$ There exist a real-valued random variable $Y$ with its d.f. $F_{Y}(x) \in \mathcal{D}$, and some positive integer $n_{0}$, positive constants $C_{1}$ and $T$ such that for all $n>n_{0}$,

$$
\frac{1}{n} \sum_{i=1}^{n} \bar{F}_{i}(x) \geq C_{1} \bar{F}_{Y}(x)
$$

holds uniformly for all $x \geq T$, and $E\left(Y^{+}\right)^{s}<\infty$ for some $s>1$.

$\left(\mathrm{A}_{2}\right)$ There exist a real-valued random variable $Z$ with its d.f. $F_{Z}(x) \in \mathcal{D}$, and positive constants $C_{2}, C_{3}$ and $T$ such that for every $n \geq 1$,

$$
\frac{1}{n} \sum_{i=1}^{n} \bar{F}_{i}(x) \leq C_{2} \bar{F}_{Z}(x)
$$

holds uniformly for all $x \geq T$, and for $\mathrm{n}$ large enough,

$$
\frac{1}{n} \sum_{i=1}^{n} F_{i}(x) \leq C_{3} F_{Z}(x)
$$

holds uniformly for all $x \leq-T$; and $E Z<\infty, J_{F_{Z}}^{-}>1$.

$\left(\mathrm{A}_{3}\right)$

$$
\limsup _{x \rightarrow \infty} \bar{F}_{Z}(x) / \bar{F}_{Y}(x)<\infty
$$

$\left(\mathrm{A}_{4}\right)$

$$
F_{Z}(-x)=o\left(\bar{F}_{Y}(x)\right)
$$

Remark 2.1 According to (2.7), (2.8), and (2.10), we can see that the r.v. $Z$ 's and $Y$ 's right tails are weak equivalent, i.e., $\bar{F}_{Z}(x) \asymp \bar{F}_{Y}(x)$. The assumption $\left(\mathrm{A}_{4}\right)$, which is equivalent to $F_{Z}(-x)=o\left(\bar{F}_{Z}(x)\right)$, shows the r.v. $Z$ 's left tails are lighter than the r.v. $Y$ 's right tails. It is clear that all assumptions $\left(\mathrm{A}_{1}\right)-\left(\mathrm{A}_{4}\right)$ are easily satisfied. 
The main results of this paper are given below.

Theorem 2.1 Let the random variables $\left\{X_{n}, n \geq 1\right\}$ introduced in Section 1 be WOD and, for some $r>1, E\left(X_{n}^{-}\right)^{r}<\infty, n \geq 1$. If the assumptions $\left(\mathrm{A}_{1}\right)-\left(\mathrm{A}_{4}\right)$ hold and there exists $a$ positive number $\beta<J_{F_{Z}}^{-}-1$ such that

$$
g_{L}(n)=o\left(n^{\beta}\right) \quad \text { and } \quad g_{U}(n)=o\left(n^{\beta}\right)
$$

then (1.1) and (1.2) hold, respectively.

Theorem 2.2 In addition to the conditions of Theorem 2.1, if one of the following two conditions is satisfied:

(I) when $c \geq 0$, we have for arbitrarily fixed $\omega>0$ and some $r>J_{F_{Y}}^{+}$

$$
E\left[N(t)^{r} \mathbf{1}_{(N(t)>(1+\omega) \lambda(t))}\right]=O(\lambda(t))
$$

(II) when $c<0$, we have for all $0<\omega<1$

$$
\lim _{t \rightarrow \infty} \frac{P_{r}(N(t) \leq(1-\omega) \lambda(t))}{\bar{F}_{Y}(\lambda(t))}=0,
$$

then (1.3) and (1.4) hold, respectively.

Remark 2.2 According to (3.13), (3.15), and (3.16), we can take

$$
M_{1}=\left(C_{1} / C_{2}\right) \liminf _{x \rightarrow \infty}\left(\bar{F}_{Y}(u x / a) / \bar{F}_{Z}(x / b)\right)
$$

and

$$
M_{2}=\left(C_{2} / C_{1}\right) \limsup _{x \rightarrow \infty}\left(\bar{F}_{Z}(v x / b) / \bar{F}_{Y}(x / a)\right)
$$

in Theorem 2.1, respectively, where $a, b, u, v, C_{1}$, and $C_{2}$ are some fixed positive constants. For the given distribution functions $\bar{F}_{Z}(x)$ and $\bar{F}_{Y}(x)$, we can obtain the sharp lower and upper bound $M_{1}, M_{2}$. Hence, though the above expressive forms are not nice-looking, causes no trouble for real applications. For $\widetilde{M}_{1}$ and $\widetilde{M}_{2}$, by the proof of Theorem 2.2, we can make a similar remark.

\section{Proof of Theorem 2.1}

We start with a series of lemmas based on which Theorem 2.1 will be proved. The proofs of the following Lemmas 3.1 and 3.2 are straightforward and are therefore omitted.

Lemma 3.1 If $E\left(X^{+}\right)^{s}<\infty$ for some $s>0$, then the relation

$$
\lim _{n \rightarrow \infty} \sup _{x \geq \gamma n} n^{s} \bar{F}(x)=0
$$

holds for arbitrarily fixed $\gamma>0$. 
Lemma 3.2 If $E\left(X_{i}^{ \pm}\right)^{q}<\infty, i \geq 1$, and $E\left(Z^{ \pm}\right)^{q}<\infty$ for some $q \geq 1$, and (2.8), (2.9) hold, then there exists positive constant $\hat{\mu}_{q}^{ \pm}<\infty$ such that

$$
\sum_{i=1}^{n} E\left(X_{i}^{ \pm}\right)^{q} \leq n \hat{\mu}_{q}^{ \pm}
$$

holds for any $n=1,2, \ldots$.

Lemma 3.3 Let $\left\{X_{n}, n \geq 1\right\}$ be a sequence of real-valued and WUOD r.v.s with $X_{n}$ 's d.f. $F_{n}(x)=1-\bar{F}_{n}(x)$ and $\mu_{n}=E X_{n}=0$ for every $n \geq 1$, and let $\left\{c_{1}, c_{2}, \ldots\right\}$ be a sequence of real numbers satisfying $0<a \leq c_{i} \leq b<\infty, i \geq 1$. If the assumptions $\left(\mathrm{A}_{1}\right)-\left(\mathrm{A}_{3}\right)$ hold and there exists a positive number $\beta>0$ such that

$$
g_{U}(n)=O\left(n^{\beta}\right)
$$

then there exists a constant $M_{2}>0$ such that the relation

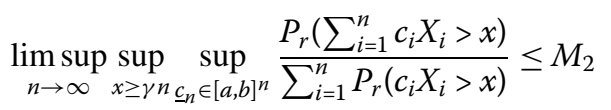

holds for arbitrarily fixed constant $\gamma>0$, where $\underline{c}_{n}=\left(c_{1}, c_{2}, \ldots, c_{n}\right)$.

Proof For arbitrarily fixed $0<v<1$, let $\widetilde{X}_{i}=\min \left\{X_{i}, \nu x / c_{i}\right\}, i \geq 1$ and $\widetilde{S}_{n}=\sum_{i=1}^{n} c_{i} \widetilde{X}_{i}$. Using the standard truncation technique, we have

$$
P_{r}\left(\sum_{i=1}^{n} c_{i} X_{i}>x\right) \leq \sum_{i=1}^{n} P_{r}\left(c_{i} X_{i}>v x\right)+P_{r}\left(\widetilde{S}_{n}>x\right)
$$

Now, we deal with the second term on the right-hand side of (3.3). Let $c=c\left(n, x, \underline{c}_{n}\right)=$ $\max \left\{-\log \sum_{k=1}^{n} P_{r}\left(c_{k} X_{k}>v x\right), 1\right\}$, then we can obtain $\lim \sup _{n \rightarrow \infty} \sup _{x \geq \gamma n} \sup _{\underline{c}_{n} \in[a, b]^{n}} c=\infty$ according to (2.8) and Lemma 3.1. Write $\mathbf{W}=P_{r}\left(\widetilde{S}_{n}>x\right) / \sum_{i=1}^{n} P_{r}\left(c_{i} X_{i}>v x\right)$. For a positive number $h=h\left(n, x, \underline{c}_{n}\right)>0$, which we shall specify later, by Chebyshev's inequality and Proposition 2.1, we have

$$
\begin{aligned}
\mathbf{W} \leq & g_{U}(n) \exp \left\{-h x+c+\sum_{i=1}^{n} \log E e^{h c_{i} \tilde{X}_{i}}\right\} \\
\leq & g_{U}(n) \exp \left\{-h x+c+\sum_{i=1}^{n} \log \left[\int_{-\infty}^{\nu x / c_{i}}\left(e^{h c_{i} y}-1\right) d F_{i}(y)\right.\right. \\
& \left.\left.+\left(e^{h v x}-1\right) P_{r}\left(c_{i} X_{i}>v x\right)+1\right]\right\} \\
\leq & g_{U}(n) \exp \left\{-h x+c+\sum_{i=1}^{n}\left[\int_{-\infty}^{v x / c_{i}}\left(e^{h c_{i} y}-1\right) d F_{i}(y)\right.\right. \\
& \left.\left.+\left(e^{h v x}-1\right) P_{r}\left(c_{i} X_{i}>v x\right)\right]\right\}
\end{aligned}
$$




$$
\begin{aligned}
\leq & g_{U}(n) \exp \left\{-h x+c+\sum_{i=1}^{n} \int_{-\infty}^{0}\left(e^{h c_{i} y}-1\right) d F_{i}(y)\right. \\
& \left.+\sum_{i=1}^{n}\left(\int_{0}^{\frac{\nu x}{c_{i} c^{k}}}+\int_{\frac{\nu x}{c_{i} c^{c^{*}}}}^{\frac{\nu x}{c_{i}}}\right)\left(e^{h c_{i} y}-1\right) d F_{i}(y)+\left(e^{h \nu x}-1\right) \sum_{i=1}^{n} P_{r}\left(c_{i} X_{i}>v x\right)\right\} \\
= & g_{U}(n) \exp \left\{-h x+c+I_{1}+I_{2}+I_{3}+\left(e^{h \nu x}-1\right) \sum_{i=1}^{n} P_{r}\left(c_{i} X_{i}>v x\right)\right\},
\end{aligned}
$$

where $\kappa>1$ is an arbitrarily fixed constant. Take $h=\left(c-\kappa p_{2} \log c\right) / \nu x$, where $p_{2}>J_{F_{Y}}^{+}$. By (2.4) and (2.7),

$$
\begin{aligned}
\limsup _{n \rightarrow \infty} \sup _{x \geq \gamma n} \sup _{\underline{c}_{n} \in[a, b]^{n}} h & \leq \limsup _{n \rightarrow \infty} \sup _{x \geq \gamma n} \frac{-\log C_{1} n \bar{F}_{Y}(\nu x / a)}{\nu x} \\
& \leq \limsup _{n \rightarrow \infty} \sup _{x \geq \gamma n} \frac{-\log C_{1} n\left(\frac{v x}{a}\right)^{-p_{2}}}{\nu x}=0 .
\end{aligned}
$$

For $I_{1}$, we have

$$
\begin{aligned}
\frac{I_{1}}{h n} & =\int_{-\infty}^{0}\left(-\frac{1}{n} \sum_{i=1}^{n} F_{i}(y) c_{i} e^{h c_{i} y}\right) d y \\
& \leq b\left(\int_{-\infty}^{-T}+\int_{-T}^{0}\right) \frac{1}{n} \sum_{i=1}^{n} F_{i}(y)\left(1-e^{h c_{i} y}\right) d y-\frac{1}{n} \sum_{i=1}^{n} c_{i} E X_{i}^{-}
\end{aligned}
$$

Denote $g\left(n, h, y, \underline{c}_{n}\right)=\left(\sum_{i=1}^{n} F_{i}(y)\left(1-e^{h c_{i} y}\right)\right) / n$ and $g_{n}(y)=\sup _{x \geq \gamma n} \sup _{\underline{c}_{n} \in[a, b]^{n}} g\left(n, h, y, \underline{c}_{n}\right)$. By (2.9), we see that there exists $N>0$ such that for $n \geq N$ and all $y \leq-T,\left|g_{n}(y)\right| \leq C_{3} F_{Z}(y)$. And for any $[s, t] \subset(-\infty,-T],\left|g_{n}(y)\right| \leq \sup _{x \geq \gamma n} \sup _{\underline{c}_{n} \in[a, b]^{n}}\left(1-e^{b h s}\right) \rightarrow 0, n \rightarrow \infty$ for all $y \in[s, t]$. Hence,

$$
\begin{aligned}
\limsup _{n \rightarrow \infty} \sup _{x \geq \gamma n \underline{\underline{c}}_{n} \in[a, b]^{n}} \int_{-\infty}^{-T} g\left(n, h, y, \underline{c}_{n}\right) d y & \leq \limsup _{n \rightarrow \infty} \int_{-\infty}^{-T} g_{n}(y) d y \\
& =\int_{-\infty}^{-T} \lim _{n \rightarrow \infty} g_{n}(y) d y=0 .
\end{aligned}
$$

From the definition of $g_{n}(y)$, we know that $\left|g_{n}(y)\right| \leq 1$ for every $n$ and all $y \in[-T, 0)$, and $g_{n}(y) \rightarrow 0, n \rightarrow \infty$ for all $y \in[-T, 0)$. So,

$$
\begin{aligned}
\limsup _{n \rightarrow \infty} \sup _{x \geq \gamma n} \sup _{\underline{\underline{c}}_{n} \in[a, b]^{n}} \int_{-T}^{0} g\left(n, h, y, \underline{c}_{n}\right) d y & \leq \limsup _{n \rightarrow \infty} \int_{-T}^{0} g_{n}(y) d y \\
& =\int_{-T}^{0} \lim _{n \rightarrow \infty} g_{n}(y) d y=0 .
\end{aligned}
$$

Combining (3.6), (3.7) with (3.5), we obtain

$$
I_{1}=\varphi n h-h \sum_{i=1}^{n} c_{i} E X_{i}^{-},
$$


where $\lim \sup _{n \rightarrow \infty} \sup _{x \geq \gamma n} \sup _{\underline{c}_{n} \in[a, b]^{n}} \varphi=0$. For $I_{2}$ and $I_{3}$, we have

$$
\begin{aligned}
I_{2}+I_{3} & \leq \sum_{i=1}^{n}\left(\int_{0}^{\frac{\nu x}{c_{i} c^{k}}} h c_{i} y e^{h c_{i} y} d F_{i}(y)+\int_{\frac{\nu x}{c_{i} c^{c^{k}}}}^{\frac{\nu x}{c_{i}}} e^{h c_{i} y} d F_{i}(y)\right) \\
& \leq \sum_{i=1}^{n}\left(h c_{i} e^{\frac{h \nu x}{c^{k}}} E X_{i}^{+}+e^{h \nu x} \bar{F}_{i}\left(\frac{\nu x}{c_{i} c^{\kappa}}\right)\right),
\end{aligned}
$$

where lim $\sup _{n \rightarrow \infty} \sup _{x \geq \gamma n} \sup _{{\underline{c_{n}}}_{n} \in[a, b]^{n}} x / c^{\kappa} \rightarrow \infty$ according to (2.4) and (2.7). Plugging (3.8) and (3.9) into (3.4) yields

$$
\begin{aligned}
\mathbf{W} \leq & g_{U}(n) \exp \left\{-h x+c+\varphi h n+h b \sum_{i=1}^{n} E X_{i}^{+}\left(e^{\frac{h \nu x}{c^{k}}}-1\right)\right. \\
& \left.+C_{2} n \bar{F}_{Z}\left(\frac{v x}{b c^{\kappa}}\right) e^{h v x}+C_{2} n \bar{F}_{Z}\left(\frac{\nu x}{b}\right)\left(e^{h \nu x}-1\right)\right\} \\
\leq & g_{U}(n) \exp \left\{-h x+c+\left[\varphi+b \hat{\mu}_{1}^{+}\left(e^{h u v / c^{\kappa}}-1\right)\right] h n\right. \\
& \left.+C_{2} \widetilde{C}_{2} c^{\kappa p_{2}} n \bar{F}_{Z}\left(\frac{v x}{b}\right) e^{h v x}+C_{2} n \bar{F}_{Z}\left(\frac{v x}{b}\right) e^{h v x}\right\} \\
\leq & g_{U}(n) \exp \left\{-h x+c+\left[\varphi+b \hat{\mu}_{1}^{+}\left(e^{h u v / c^{\kappa}}-1\right)\right] h n\right. \\
& \left.+\hat{B}\left(c^{\kappa p_{2}}+1\right) n \bar{F}_{Z}\left(\frac{\nu x}{b}\right) e^{h v x}\right\}
\end{aligned}
$$

where in the first step we apply (2.8), in the second step we use Lemma 3.2 and (2.2), and $\hat{B}$ is an appropriately chosen positive number. By the value of $h$, we have

$$
\limsup _{n \rightarrow \infty} \sup _{x \geq \gamma n} \sup _{\underline{c}_{n} \in[a, b]^{n}} \hat{B}\left(c^{\kappa p_{2}}+1\right) n \bar{F}_{Z}\left(\frac{\nu x}{b}\right) e^{h \nu x}<\infty
$$

and

$$
\limsup _{n \rightarrow \infty} \sup _{x \geq \gamma n \underline{c}_{n} \in[a, b]^{n}} \frac{\left[\varphi+b \hat{\mu}_{1}^{+}\left(e^{h u v / c^{k}}-1\right)\right] h n+\left(\kappa p_{2} \log c\right) / v}{c}=0 .
$$

Hence,

$$
\mathbf{W} \leq g_{U}(n) n^{-\beta} \exp \left\{\beta \log n+\left(1-\frac{1}{v}\right) c+o(c)+O(1)\right\} .
$$

Using (2.3) and (2.8), we obtain

$$
\limsup _{n \rightarrow \infty} \sup _{x \geq \gamma n} \sup _{\underline{c}_{n} \in[a, b]^{n}} \frac{\log n}{c} \leq \frac{1}{p_{1}-1}
$$


where $1<p_{1}<J_{F_{Z}}^{-}$. Taking $0<v<\left(p_{1}-1\right) /\left(\beta+p_{1}-1\right)<1$, from (3.11), we have

$$
\begin{aligned}
\limsup \sup _{n \rightarrow \infty} \sup _{x \geq \gamma n_{\underline{c}_{n}} \in[a, b]^{n}} \mathbf{W} \leq & \limsup _{n \rightarrow \infty} \sup _{x \geq \gamma n} \sup _{\underline{c}_{n} \in[a, b]^{n}} g_{U}(n) n^{-\beta} \exp \left\{\left(\frac{\beta}{p_{1}-1}+1-\frac{1}{v}\right) c\right. \\
& +o(c)+O(1)\}=0 .
\end{aligned}
$$

Applying (2.7), (2.8), and (2.10), we know that there exists $M_{2}>0$ such that

$$
\limsup \sup _{n \rightarrow \infty} \sup _{x \geq \gamma^{n} \underline{c}_{n} \in[a, b]^{n}} \frac{\sum_{i=1}^{n} P_{r}\left(c_{i} X_{i}>v x\right)}{\sum_{i=1}^{n} P_{r}\left(c_{i} X_{i}>x\right)} \leq M_{2} .
$$

Combining (3.12), (3.13) with (3.3), we can obtain (3.2).

Lemma 3.4 Let $\left\{X_{n}, n \geq 1\right\}$ be a sequence of real-valued and WOD r.v.s with $X_{n}$ 's d.f. $F_{n}(x)=1-\bar{F}_{n}(x)$ and $\mu_{n}=E X_{n}=0$ for every $n \geq 1$, and let $\left\{c_{1}, c_{2}, \ldots\right\}$ be a sequence of real numbers satisfying $0<a \leq c_{i} \leq b<\infty, i \geq 1$. If the assumptions $\left(\mathrm{A}_{1}\right)-\left(\mathrm{A}_{4}\right)$ hold and there exists a positive number $\beta<J_{F_{Z}}^{-}-1$ such that

$$
g_{U}(n)=o\left(n^{\beta}\right) \text { and } g_{L}(n)=o\left(n^{\beta}\right)
$$

then there exists a constant $M_{1}>0$ such that the relation

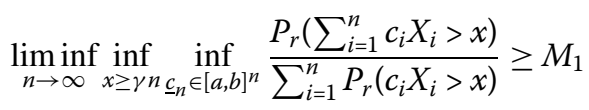

holds for arbitrarily fixed constant $\gamma>0$, where $\underline{c}_{n}=\left(c_{1}, c_{2}, \ldots, c_{n}\right)$.

Proof It is sufficient to prove that

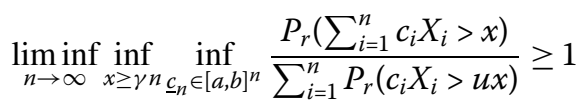

holds for arbitrarily fixed $u>1$. We write $A_{k}=\left\{c_{k} X_{k}>u x\right\}$ and $B_{k}=\bigcap_{1 \leq i \neq k \leq n} A_{i}^{c}$. Observing that $A_{k} \cap B_{k}, k=1,2, \ldots, n$, are mutually disjoint, we have

$$
\begin{aligned}
P_{r}\left(\sum_{i=1}^{n} c_{i} X_{i}>x\right) \geq & \sum_{k=1}^{n}\left[P_{r}\left(A_{k}\right)-P_{r}\left(A_{k} \cap B_{k}^{c}\right)-P_{r}\left(\sum_{i=1}^{n} c_{i} X_{i} \leq x, A_{k} \cap B_{k}\right)\right] \\
\geq & \sum_{k=1}^{n}\left[P_{r}\left(A_{k}\right)-\sum_{1 \leq i \neq k \leq n} P_{r}\left(A_{k} \cap A_{i}\right)\right. \\
& \left.-P_{r}\left(\sum_{i: 1 \leq i \leq n, i \neq k} c_{i} X_{i} \leq(1-u) x, A_{k} \cap B_{k}\right)\right] \\
\geq & \sum_{k=1}^{n} P_{r}\left(A_{k}\right)\left[1-g_{U}(n) \sum_{k=1}^{n} P_{r}\left(A_{k}\right)\right] \\
& -\sum_{k=1}^{n} P_{r}\left(\sum_{i: 1 \leq i \leq n, i \neq k} c_{i} X_{i} \leq(1-u) x, A_{k} \cap B_{k}\right)=J_{1}-J_{2},
\end{aligned}
$$

where at the third step we used Proposition 2.1. 
For $J_{1}$, by (2.3), (2.8), and (3.14), we have for arbitrarily fixed $\gamma>0$

$$
\begin{aligned}
& \liminf _{n \rightarrow \infty} \inf _{x \geq \gamma n} \inf _{\underline{c}_{n} \in[a, b]^{n}} \frac{J_{1}}{\sum_{k=1}^{n} P_{r}\left(c_{k} X_{k}>u x\right)} \\
& \geq 1-\limsup _{n \rightarrow \infty} \sup _{x \geq \gamma n} g_{U}(n) n C_{2} \bar{F}_{Z}\left(\frac{u x}{b}\right) \\
& \geq 1-\limsup _{n \rightarrow \infty} C_{2} g_{U}(n) n^{1-p_{1}}\left(\frac{b}{u \gamma}\right)^{p_{1}}=1,
\end{aligned}
$$

where $p_{1}=\beta+1$.

Now we deal with $J_{2}$. For fixed $0<w<1$, Let $W_{k}=-X_{k}$ and $\widetilde{W}_{k}=\min \left\{W_{k},(w x) / c_{k}\right\}$, then

$$
\begin{aligned}
J_{2} \leq & \sum_{k=1}^{n} P_{r}\left(A_{k} \cap B_{k}, \bigcup_{i=1}^{n}\left\{c_{i} W_{i}>w x\right\}\right) \\
& +\sum_{k=1}^{n} P_{r}\left(\sum_{i: 1 \leq i \leq n, i \neq k} c_{i} X_{i} \leq(1-u) x, \bigcap_{j=1}^{n}\left\{c_{j} W_{j} \leq w x\right\}\right) \\
\leq & \sum_{i=1}^{n} P_{r}\left(c_{i} W_{i}>w x\right)+\sum_{k=1}^{n} P_{r}\left(c_{k} \widetilde{W}_{k} \geq(u-1) x\right)=K_{1}+K_{2} .
\end{aligned}
$$

For $K_{1}$, by (2.7), (2.9)-(2.11), we have

$$
\limsup _{n \rightarrow \infty} \sup _{x \geq \gamma^{n} \underline{c}_{n} \in[a, b]^{n}} \sup _{\sum_{i=1}^{n} P_{r}\left(c_{i} X_{i}>u x\right)} \leq \limsup _{x \rightarrow \infty} \frac{C_{3} F_{Z}\left(-\frac{w}{b} x\right)}{C_{1} \bar{F}_{Y}\left(\frac{u}{a} x\right)}=0 .
$$

For $K_{2}$, using Chebyshev's inequality and Proposition 2.1 again, we have

$$
\begin{aligned}
K_{2} \leq & \sum_{k=1}^{n} g_{L}(n-1) \exp \left\{-h(u-1) x+\sum_{i: 1 \leq i \leq n, i \neq k} \log E e^{h c_{i} \widetilde{W}_{i}}\right\} \\
\leq & \sum_{k=1}^{n} g_{L}(n-1) \exp \{-h(u-1) x \\
& \left.+\sum_{i: 1 \leq i \leq n, i \neq k}\left[\int_{-\infty}^{\frac{w x}{c_{i}}}\left(e^{h c_{i} y}-1\right) d F_{W_{i}}(y)+\left(e^{h w x}-1\right) P_{r}\left(c_{i} W_{i}>w x\right)\right]\right\} \\
\leq & \sum_{k=1}^{n} g_{L}(n-1) \exp \left\{-h(u-1) x+\sum_{i: 1 \leq i \leq n, i \neq k}\left[\int_{-\infty}^{0}\left(e^{h c_{i} y}-1\right) d F_{W_{i}}(y)\right.\right. \\
& +\int_{0}^{\frac{w x}{c_{i}}} \frac{e^{h c_{i} y}-1-h c_{i} y}{\left(c_{i} y\right)^{s}}\left(c_{i} y\right)^{s} d F_{W_{i}}(y)+h c_{i} E X_{i}^{-} \\
& \left.\left.+\left(e^{h w x}-1\right) P_{r}\left(c_{i} W_{i}>w x\right)\right]\right\}
\end{aligned}
$$

where $h=\left(\log \left(w^{s-1} x^{s} / n \hat{\mu}_{s}^{-}+1\right)\right) / w x$ and $s>1$. Using similar techniques as in (3.8), we can obtain

$$
\left|\sum_{i: 1 \leq i \leq n, i \neq k}\left[\int_{-\infty}^{0}\left(e^{h c_{i} y}-1\right) d F_{W_{i}}(y)+h c_{i} E X_{i}^{-}\right]\right| \leq|\alpha| n h,
$$


where $\alpha \rightarrow 0$ holds uniformly for $x \geq \gamma n$, as $n \rightarrow \infty$. By (2.9) and (2.11),

$$
\begin{aligned}
& \limsup _{n \rightarrow \infty} \sup _{x \geq \gamma n} \sup _{\underline{c}_{n} \in[a, b]^{n}} \sum_{i: 1 \leq i \leq n, i \neq k}\left(e^{h w x}-1\right) P_{r}\left(c_{i} W_{i}>w x\right) \\
& \quad \leq \limsup _{x \rightarrow \infty} \frac{w^{s-1}}{\hat{\mu}_{s}^{-}} C_{3} x^{s} \bar{F}_{Y}\left(\frac{b}{w} x\right) \rightarrow 0 .
\end{aligned}
$$

Take sufficiently large $n$ such that $|\alpha| \leq(u-1) \gamma / 2$. Combining (3.21)-(3.23) and observing the monotonicity of $0 \leq\left(e^{h c_{i} y}-1-h c_{i} y\right) /\left(c_{i} y\right)^{s}$ for all $y>0$, we have

$$
\begin{aligned}
K_{2} \leq & \sum_{k=1}^{n} g_{L}(n-1) n^{-\beta} \exp \{\beta \log n-h(u-1) x+|\alpha| n h \\
& \left.+\frac{e^{h w x}-1-h w x}{(w x)^{s}} \sum_{i: 1 \leq i \leq n, i \neq k} c_{i}^{s} E\left(X_{i}^{-}\right)^{s}+o(1)\right\} \\
\leq & \sum_{k=1}^{n} g_{L}(n-1) n^{-\beta} \exp \left\{\log \left(\frac{x}{\gamma}\right)^{\beta}+\log \left(\frac{w^{s-1} x^{s}}{n \hat{\mu}_{s}^{-}}+1\right)^{-\frac{u-1}{2 w}}\right. \\
& \left.+\frac{e^{h w x}-1}{(w x)^{s}} b^{s} n \hat{\mu}_{s}^{-}+o(1)\right\} \leq \widetilde{C} n x^{\beta-\frac{(s-1)(u-1)}{2 w}},
\end{aligned}
$$

where $\widetilde{C}$ is some positive constant. For fixed $u>1$, we take $0<w<1$ such that $\frac{(s-1)(u-1)}{2 w}>$ $\beta+J_{F_{Y}}^{+}$. By (2.4) and (2.7), we have

$$
\limsup _{n \rightarrow \infty} \sup _{x \geq \gamma n_{\underline{C}_{n}} \in[a, b]^{n}} \sup _{\sum_{k=1}^{n} P_{r}\left(c_{k} X_{k}>u x\right)} \leq \limsup _{x \rightarrow \infty} \frac{\widetilde{C} x^{\beta-\frac{(s-1)(u-1)}{2 w}}}{C_{1} \bar{F}_{Y}\left(\frac{u}{a} x\right)}=0 .
$$

Combing (3.17)-(3.20) with (3.25), we can obtain (3.16). This ends the proof of Lemma 3.4.

Proof of Theorem 2.1 By Lemma 3.3, for arbitrarily fixed $\gamma>0$, we have uniformly for $x \geq \gamma n$

$$
\begin{aligned}
P_{r}\left(\sum_{k=1}^{n} \theta_{k} X_{k}>x\right) & =E\left[P_{r}\left(\sum_{k=1}^{n} \theta_{k} X_{k}>x \mid \theta_{1}, \theta_{2}, \ldots, \theta_{n}\right)\right] \\
& \lesssim M_{2} E\left[\sum_{k=1}^{n} P_{r}\left(\theta_{k} X_{k}>x \mid \theta_{1}, \theta_{2}, \ldots, \theta_{n}\right)\right] \\
& =M_{2} \sum_{k=1}^{n} P_{r}\left(\theta_{k} X_{k}>x\right) .
\end{aligned}
$$

According to Lemma 3.4 and using a similar method of proof as in (3.26), we can obtain the remainder of Theorem 2.1.

\section{Proof of Theorem 2.2}

For proving Theorems 2.2, we first give two lemmas. 
Lemma 4.1 Let $\left\{X_{n}, n \geq 1\right\}$ be a sequence of real-valued and WUOD r.v.s with $X_{n}$ 's d.f. $F_{n}(x)=1-\bar{F}_{n}(x)$ and $0<E X_{n}^{+}<0$ for every $n \geq 1$, and Let $\left\{\theta_{i}, i \geq 1\right\}$ be a sequence of nonnegative r.v.s satisfying $P_{r}\left(a \leq \theta_{i} \leq b\right)=1, i \geq 1,0<a \leq b<\infty$ and independent of $\left\{X_{n}, n \geq\right.$ 1\}. If (2.8) and $E Z^{+}<\infty$ hold and $g_{U}(n)=O\left(n^{\beta}\right)$ for some positive number $\beta$, then for every fixed $u>0$, there is $\hat{D}=\hat{D}(u)>0$ such that

$$
P_{r}\left(\sum_{i=1}^{n} \theta_{i} X_{i}>x\right) \leq \sum_{i=1}^{n} P_{r}\left(\theta_{i} X_{i}>u x\right)+\hat{D}\left(\frac{n}{x}\right)^{\frac{1}{u}} n^{\beta}
$$

holds for large $n$ and all $x>0$.

Proof Using the techniques similar to Lemma 3.3 with some obvious modifications, we can prove the lemma.

Combining Lemma 2.1 of Chen et al. [6] with Lemma 3.1 of $\mathrm{Ng}$ et al. [11], we can obtain the following lemma.

Lemma 4.2 If a non-negative random process $\{\zeta(t), t \geq 0\}$ satisfies $E \zeta(t) \rightarrow 1, t \rightarrow \infty$, then (i)-(iv) are mutually equivalent:

(i) $\zeta(t) \stackrel{P_{r}}{\rightarrow} 1$, as $t \rightarrow \infty$;

(ii) for every fixed $\theta>0, E \zeta(t) \mathbf{1}_{\{\zeta(t)-1>\theta\}}=o(1)$;

(iii) for every fixed $\theta>0, E \zeta(t) \mathbf{1}_{\{\zeta(t)-1 \mid>\theta\}}=o(1)$;

(iv) for every fixed $0<\theta<1, P_{r}(1-\zeta(t) \geq \theta)=o(1)$.

By Lemma 4.2 and (2.13), we know that

$$
\frac{N(t)}{\lambda(t)} \stackrel{P_{r}}{\rightarrow} 1 .
$$

Proof of Theorem 2.2 Now, we prove Theorem 2.2 under condition (I). Using Theorem 2.1 and (2.8), we obtain for any fixed $\sigma>0$ the result that there exists a positive integral number $N$ such that when $n \geq N$, for sufficiently large $x$,

$$
P_{r}\left(\sum_{i=1}^{n} \theta_{i} X_{i}>x\right) \leq\left(M_{2}+\sigma\right) \sum_{i=1}^{n} P_{r}\left(\theta_{i} X_{i}>x\right) \leq\left(M_{2}+\sigma\right) C_{2} n \bar{F}_{Z}\left(\frac{x}{b}\right) .
$$

It is clear that for every $n=1,2, \ldots, N$ and all large $x$,

$$
P_{r}\left(\sum_{i=1}^{n} \theta_{i} X_{i}>x\right) \leq \sum_{i=1}^{n} P_{r}\left(\theta_{i} X_{i}>x / N\right) \leq C_{2} n \bar{F}_{Z}\left(\frac{x}{N b}\right) .
$$

Hence, by (4.3) and (4.4), there exists some positive number $D$, for every $n=1,2, \ldots$, and all sufficiently large $x$,

$$
P_{r}\left(\sum_{i=1}^{n} \theta_{i} X_{i}>x\right) \leq \operatorname{Dn} \bar{F}_{Z}(x)
$$


Take $0<\omega<1$ such that $c(1+\omega)<\gamma$. Throughout this proof, we suppose that $x \in$ $[\gamma \lambda(t), \infty)$. Consider the following decomposition:

$$
\begin{aligned}
P_{r}\left(S_{c}^{\theta}(t)>x\right) & =\left(\sum_{n<(1-\omega) \lambda(t)}+\sum_{(1-\omega) \lambda(t) \leq n \leq(1+\omega) \lambda(t)}+\sum_{n>(1+\omega) \lambda(t)}\right) P_{r}\left(S_{n}^{\theta}>x-n c\right) P_{r}(N(t)=n) \\
& =L_{1}+L_{2}+L_{3} .
\end{aligned}
$$

Firstly, we deal with $L_{1}$. For sufficiently large $t$, by (4.5), we have

$$
\begin{aligned}
L_{1} & \leq \sum_{n<(1-\omega) \lambda(t)} P_{r}\left(S_{n}^{\theta}>x-(1-\omega) c \lambda(t)\right) P_{r}(N(t)=n) \\
& \leq D \bar{F}_{Z}(x-(1-\omega) c \lambda(t)) \sum_{n<(1-\omega) \lambda(t)} n P_{r}(N(t)=n) .
\end{aligned}
$$

For convenience, write $H=E\left[\sum_{i=1}^{N(t)} P_{r}\left(\theta_{i} X_{i}>x-c \lambda(t)\right)\right]$. According to $x-(1-\omega) c \lambda(t) \asymp$ $x-c \lambda(t),(2.7),(2.10)$, and (iv) of Lemma 4.2, we have

$$
\begin{aligned}
& \limsup _{t \rightarrow \infty} \sup _{x \geq \gamma \lambda(t)} \frac{L_{1}}{H} \\
& \quad \leq \limsup _{t \rightarrow \infty} \sup _{x \geq \gamma \lambda(t)} \frac{D \bar{F}_{Z}(x-(1-\omega) c \lambda(t)) \sum_{n<(1-\omega) \lambda(t)} n P_{r}(N(t)=n)}{C_{1} \bar{F}_{Y}\left(\frac{x-c \lambda(t)}{a}\right) E\left[N(t) \mathbf{1}_{\left\{N(t)>n_{0}\right\}}\right]} \\
& \quad \leq \widetilde{D} \limsup _{t \rightarrow \infty} \frac{\sum_{n<(1-\omega) \lambda(t)} n P_{r}(N(t)=n)}{E N(t)-n_{0}} \leq \widetilde{D} \limsup _{t \rightarrow \infty} \frac{E\left[\frac{N(t)}{\lambda(t)} \mathbf{1}_{\{N(t)<(1-\omega) \lambda(t)\}}\right]}{1-\frac{n_{0}}{\lambda(t)}} \\
& \quad \leq \widetilde{D}(1-\omega) \lim _{t \rightarrow \infty} P_{r}\left(\frac{N(t)}{\lambda(t)}<1-\omega\right)=0,
\end{aligned}
$$

where $\widetilde{D}$ is some positive constant.

Secondly, we deal with $L_{2}$. On the one hand, by Theorem 2.1, for arbitrary $\varepsilon_{1}>0$ and sufficiently large $t$,

$$
\begin{aligned}
L_{2} & \geq\left(M_{1}-\varepsilon_{1}\right) \sum_{(1-\omega) \lambda(t) \leq n \leq(1+\omega) \lambda(t)} \sum_{i=1}^{n} P_{r}\left(\theta_{i} X_{i}>x-n c\right) P_{r}(N(t)=n) \\
& \geq\left(M_{1}-\varepsilon_{1}\right) C_{1} \bar{F}_{Y}\left(\frac{x-(1-\omega) c \lambda(t)}{a}\right) \sum_{(1-\omega) \lambda(t) \leq n \leq(1+\omega) \lambda(t)} n P_{r}(N(t)=n) .
\end{aligned}
$$

By (2.8), we have $H \leq C_{2} \bar{F}_{Z}((x-c \lambda(t)) / b) \lambda(t)$. Using (2.10) and (iii) of Lemma 4.2, we know that there is a positive constant $\widetilde{M}_{1}$ such that

$$
\liminf _{t \rightarrow \infty} \inf _{x \geq \gamma \lambda(t)} \frac{L_{2}}{H} \geq \tilde{M}_{1} \lim _{t \rightarrow \infty} E\left[\frac{N(t)}{\lambda(t)} \mathbf{1}_{\left\{\left|\frac{N(t)}{\lambda(t)}-1\right| \leq \omega\right\}}\right]=\tilde{M}_{1}
$$

On the other hand, using similar techniques as in (4.8), for any $\varepsilon_{2}>0$ and sufficiently large $t$, we have

$$
L_{2} \leq\left(M_{2}+\varepsilon_{2}\right) C_{2} \bar{F}_{Z}\left(\frac{x-(1+\omega) c \lambda(t)}{b}\right) \sum_{(1-\omega) \lambda(t) \leq n \leq(1+\omega) \lambda(t)} n P_{r}(N(t)=n) .
$$


By (2.7), we have

$$
H \geq C_{1} \bar{F}_{Y}\left(\frac{x-c \lambda(t)}{a}\right) E\left[N(t) \mathbf{1}_{\left\{N(t)>n_{0}\right\}}\right]
$$

Then, by (4.10) and (4.11), there exists a positive number $\widetilde{M}_{2}$ such that

$$
\limsup _{t \rightarrow \infty} \sup _{x \geq \gamma \lambda(t)} \frac{L_{2}}{H} \leq \tilde{M}_{2} \lim _{t \rightarrow \infty} \frac{E\left[\frac{N(t)}{\lambda(t)} \mathbf{1}_{\left\{\left|\frac{N(t)}{\lambda(t)}-1\right| \leq \omega\right\}}\right]}{1-\frac{n_{0}}{\lambda(t)}}=\tilde{M}_{2} .
$$

Finally, we deal with $L_{3}$. Taking $0<v<1$ and splitting $L_{3}$ into two parts, we obtain

$$
\begin{aligned}
L_{3} & =\left(\sum_{(1+\omega) \lambda(t)<n \leq(1-v) x / c}+\sum_{n>\max \{(1+\omega) \lambda(t),(1-v) x / c\}}\right) P_{r}\left(\sum_{i=1}^{n} \theta_{i} X_{i}>x-n c\right) P_{r}(N(t)=n) \\
& =R_{1}+R_{2},
\end{aligned}
$$

where $R_{1}$ is understood as 0 in case $(1+\omega) \lambda(t)>(1-v) x / c$. For $R_{1}$, taking $u=1 / p$ in (4.1) and letting $p>J_{F_{Y}}^{+}$, we have

$$
\begin{aligned}
R_{1} & \leq \sum_{(1+\omega) \lambda(t)<n \leq(1-v) x / c} P_{r}\left(\sum_{i=1}^{n} \theta_{i} X_{i}>v x\right) P_{r}(N(t)=n) \\
& \leq \sum_{(1+\omega) \lambda(t)<n \leq(1-v) x / c}\left(\sum_{i=1}^{n} P_{r}\left(\theta_{i} X_{i}>v x / p\right)+\hat{D}(v x)^{-p} n^{p+\beta}\right) P_{r}(N(t)=n) \\
& \leq C_{2} \bar{F}_{Z}\left(\frac{v x}{b p}\right) E\left[N(t) \mathbf{1}_{\{N(t)>(1+\omega) \lambda(t)\}}\right]+\hat{D}(v x)^{-p} E\left[N(t)^{p+\beta} \mathbf{1}_{\{N(t)>(1+\omega) \lambda(t)\}]} .\right.
\end{aligned}
$$

Hence, according to (ii) of Lemma 4.2, (2.13), and (2.4), we have

$$
\begin{aligned}
& \limsup _{t \rightarrow \infty} \sup _{x \geq \gamma \lambda(t)} \frac{R_{1}}{H} \\
& \leq \limsup _{t \rightarrow \infty} \sup _{x \geq \gamma \lambda(t)} \frac{C_{2} \bar{F}_{Z}\left(\frac{v x}{b p}\right) E\left[\frac{N(t)}{\lambda(t)} \mathbf{1}_{\{N(t)>(1+\omega) \lambda(t)\}}\right.}{C_{1} \bar{F}_{Y}\left(\frac{x-c \lambda(t)}{a}\right)\left(1-\frac{n_{0}}{\lambda(t)}\right)} \\
& \quad+\limsup _{t \rightarrow \infty} \sup _{x \geq \gamma \lambda(t)} \frac{\hat{D}(v x)^{-p}}{C_{1} \bar{F}_{Y}\left(\frac{x-c \lambda(t)}{a}\right)} \times \limsup _{t \rightarrow \infty} \sup _{x \geq \gamma \lambda(t)} \frac{E\left[N(t)^{p+\beta} \mathbf{1}_{\{N(t)>(1+\omega) \lambda(t)\}}\right] / \lambda(t)}{\left(1-\frac{n_{0}}{\lambda(t)}\right)} \\
& =0 .
\end{aligned}
$$

For $R_{2}$, we have

$$
\begin{aligned}
& \limsup _{t \rightarrow \infty} \sup _{x \geq \gamma \lambda(t)} \frac{R_{2}}{H} \\
& \leq \limsup _{t \rightarrow \infty} \sup _{x \geq \gamma \lambda(t)} \frac{\sum_{n>\max \{(1+\omega) \lambda(t),(1-v) x / c\}} \frac{n^{p}}{((1-v) x / c)^{p}} P_{r}(N(t)=n)}{C_{1} \bar{F}_{Y}\left(\frac{x-c \lambda(t)}{a}\right) E\left[N(t) \mathbf{1}_{\left\{N(t)>n_{0}\right\}}\right]} \\
& \leq \limsup _{t \rightarrow \infty} \sup _{x \geq \gamma \lambda(t)} \frac{((1-v) x / c)^{-p} E\left[N(t)^{p} \mathbf{1}_{\{N(t)>(1+\omega) \lambda(t)\}}\right] / \lambda(t)}{C_{1} \bar{F}_{Y}\left(\frac{x-c \lambda(t)}{a}\right)\left(1-\frac{n_{0}}{\lambda(t)}\right)}=0 .
\end{aligned}
$$


Combing (4.6), (4.7), (4.9), (4.12), (4.13), and (4.15) with (4.16), we finish the proof under condition (I).

Finally, we prove Theorem 2.2 under condition (II). Without loss of generality, we assume $c<\gamma<0$. We still take $0<\omega<1$ such that $c(1+\omega)<\gamma<0$ and use the decomposition (4.6). For $L_{1}$, we take $\gamma_{1}>0$ and divide the interval $[\gamma \lambda(t), \infty)$ into two parts, which are $\left[\gamma \lambda(t), \gamma_{1} \lambda(t)\right)$ and $\left[\gamma_{1} \lambda(t), \infty\right)$. When $x \in\left[\gamma_{1} \lambda(t), \infty\right)$, we have

$$
\bar{F}_{Z}(x-c \lambda(t)) \geq \bar{F}_{Z}\left(\left(1-c / \gamma_{1}\right) x\right) \asymp \bar{F}_{Z}(x) .
$$

When $x \in\left[\gamma \lambda(t), \gamma_{1} \lambda(t)\right)$, we have

$$
\bar{F}_{Y}(x-c \lambda(t)) \geq \bar{F}_{Y}\left(\left(\gamma_{1}-c\right) \lambda(t)\right) \asymp \bar{F}_{Y}(\lambda(t)) .
$$

Applying (2.14), (4.5), (4.17), and (4.18), we obtain

$$
\begin{aligned}
& \limsup _{t \rightarrow \infty} \sup _{x \geq \gamma \lambda(t)} \frac{L_{1}}{H} \\
& \quad \leq \limsup _{t \rightarrow \infty} \sup _{x \geq \gamma_{1} \lambda(t)} \frac{D(1-\omega) \bar{F}_{Z}(x) P_{r}(N(t) \leq(1-\omega) \lambda(t))}{C_{1} \bar{F}_{Y}\left(\frac{x-c \lambda(t)}{a}\right)\left(1-\frac{n_{0}}{\lambda(t)}\right)} \\
& \quad+\limsup _{t \rightarrow \infty} \sup _{\gamma \lambda(t) \leq x<\gamma_{1} \lambda(t)} \frac{D(1-\omega) P_{r}(N(t) \leq(1-\omega) \lambda(t))}{C_{1} \bar{F}_{Y}\left(\frac{x-c \lambda(t)}{a}\right)\left(1-\frac{n_{0}}{\lambda(t)}\right)}=0 .
\end{aligned}
$$

For $L_{3}$, since $x-c n \geq \gamma \lambda(t)-c n \geq\left(\gamma(1+\omega)^{-1}-c\right) n$, then according to Theorem 2.1, (2.10), and Lemma 4.2, we have

$$
\begin{aligned}
& \limsup _{t \rightarrow \infty} \sup _{x \geq \gamma \lambda(t)} \frac{L_{3}}{H} \\
& \quad \leq \limsup \sup _{t \rightarrow \infty} \frac{M_{2} \sum_{n \geq \gamma \lambda(1+\omega) \lambda(t)} \sum_{i=1}^{n} P_{r}\left(\theta_{i} X_{i}>x-c n\right) P_{r}(N(t)=n)}{C_{1} \bar{F}_{Y}\left(\frac{x-c \lambda(t)}{a}\right) E\left[N(t) \mathbf{1}_{\left\{N(t)>n_{0}\right\}}\right]} \\
& \quad \leq \limsup \sup _{t \rightarrow \infty} \frac{M_{2} C_{2} \bar{F}_{Z}\left(\frac{x-c \lambda(t)}{b}\right) E\left[\frac{N(t)}{\lambda(t)} \mathbf{1}_{\{N(t)>(1+\omega) \lambda(t)\}}\right]}{C_{1} \bar{F}_{Y}\left(\frac{x-c \lambda(t)}{a}\right)\left(1-\frac{n_{0}}{\lambda(t)}\right)}=0 .
\end{aligned}
$$

For $L_{2}$, observing $\lambda(t) \leq(\gamma-c)^{-1}(x-c \lambda(t))$ and $c<0$, by Theorem 2.1, (2.14), and (iii) of Lemma 4.2 , we know that, on the one hand, there exists a positive number $\widetilde{M}_{1}$ such that

$$
\begin{aligned}
& \liminf _{t \rightarrow \infty} \inf _{x \geq \gamma \lambda(t)} \frac{L_{2}}{H} \\
& \geq \liminf _{t \rightarrow \infty} \inf _{x \geq \gamma \lambda(t)} \frac{M_{1} \sum_{(1-\omega) \lambda(t) \leq n \leq(1+\omega) \lambda(t)} \sum_{i=1}^{n} P_{r}\left(\theta_{i} X_{i}>x-c n\right) P_{r}(N(t)=n)}{C_{2} \bar{F}_{Z}\left(\frac{x-c \lambda(t)}{b}\right) \lambda(t)} \\
& \geq \liminf _{t \rightarrow \infty} \inf _{x \geq \gamma \lambda(t)} \frac{M_{1} C_{1} \bar{F}_{Y}\left(\frac{x-c(1+\omega) \lambda(t)}{a}\right) E\left[\frac{N(t)}{\lambda(t)} \mathbf{1}_{\left\{\left|\frac{N(t)}{\lambda(t)}-1\right| \leq \omega\right\}}\right]}{C_{2} \bar{F}_{Z}\left(\frac{x-c \lambda(t)}{b}\right)} \\
& \geq \liminf _{t \rightarrow \infty} \inf _{x \geq \gamma \lambda(t)} \frac{M_{1} C_{1} \bar{F}_{Y}\left(a^{-1}\left(1-c \omega(\gamma-c)^{-1}\right)(x-c \lambda(t))\right) E\left[\frac{N(t)}{\lambda(t)} \mathbf{1}_{\left\{\left|\frac{N(t)}{\lambda(t)}-1\right| \leq \omega\right\}}\right]}{C_{2} \bar{F}_{Z}\left(\frac{x-c \lambda(t)}{b}\right)} \\
& \geq \tilde{M}_{1} ;
\end{aligned}
$$


on the other hand, there exists a positive number $\widetilde{M}_{2}$ such that

$$
\begin{aligned}
& \limsup _{t \rightarrow \infty} \sup _{x \geq \gamma \lambda(t)} \frac{L_{2}}{H} \\
& \quad \leq \limsup _{t \rightarrow \infty} \sup _{x \geq \gamma \lambda(t)} \frac{M_{2} C_{2} \bar{F}_{Z}\left(b^{-1}\left(1+c \omega(\gamma-c)^{-1}\right)(x-c \lambda(t))\right) E\left[\frac{N(t)}{\lambda(t)} \mathbf{1}_{\left\{\left|\frac{N(t)}{\lambda(t)}-1\right| \leq \omega\right\}}\right]}{C_{1} \bar{F}_{Y}\left(\frac{x-c \lambda(t)}{a}\right)\left(1-\frac{n_{0}}{\lambda(t)}\right)} \\
& \leq \widetilde{M}_{2} .
\end{aligned}
$$

Combing (4.19)-(4.22), we finish the proof under condition (II).

\section{Competing interests}

The authors declare that they have no competing interests.

\section{Authors' contributions}

All authors read and approved the final manuscript.

\section{Author details}

'School of Science, Dalian Nationalities University, Liaohe West Road, Dalian, 116600, China. ${ }^{2}$ School of Mathematical Sciences, Dalian University of Technology, Lingong Road, Dalian, 116024, China. ${ }^{3}$ School of Mathematical Sciences, Capital Normal University, Xisanhuan North Road, Beijing, 100048, China.

\section{Acknowledgements}

The authors would like to thank the two referees for careful reading of our manuscript and for helpful and valuable comments and suggestions, which helped us improve the earlier version of the paper. The research of the authors was supported by the Natural Science Foundation of the Inner Mongolia Autonomous Region (No. 2013MS0101), the National Natural Sciences Foundation of China (No. 11201317), and the Beijing Municipal Education Commission Foundation (No. KM201210028005).

Received: 11 May 2013 Accepted: 25 March 2014 Published: 04 Apr 2014

\section{References}

1. Embrechts, P, Klüppelberg, C, Mikosch, T: Modeling Extremal Events for Insurance and Finance. Springer, Berlin (1997)

2. Klüppelberg, C, Mikosch, T: Large deviations of heavy-tailed random sums with applications in insurance and finance. J. Appl. Probab. 34, 293-308 (1997)

3. Mikosch, T, Nagaev, AV: Large deviations of heavy-tailed sums with applications in insurance. Extremes 1, 81-110 (1998)

4. Tang, Q: Insensitivity to negative dependence of the asymptotic behavior of precise large deviations. Electron. J. Probab. 11, 107-120 (2006)

5. Liu, L: Precise large deviations for dependent random variables with heavy tails. Stat. Probab. Lett. 79, 1290-1298 (2009)

6. Chen, YQ, Yuen, KC, Ng, KW: Precise large deviations of random sums in presence of negative dependence and consistent variation. Methodol. Comput. Appl. Probab. 13, 821-833 (2011)

7. Shen, $X, L i n, Z$ : Precise large deviations for randomly weighted sums of negatively dependent random variables with consistently varying tails. Stat. Probab. Lett. 78, 3222-3229 (2008)

8. Liu, Y, Hu, Y: Large deviations for heavy-tailed random sums of independent random variables with dominatedly varying tails. Sci. China Ser. A 46, 383-395 (2003)

9. Tang, Q, Tsitsiashvili, G: Precise estimates for the ruin probability infinite horizon in a discrete time model with heavy-tailed insurance and financial risks. Stoch. Process. Appl. 108, 299-325 (2003)

10. Wang, KY, Wang, YB, Gao, QW: Uniform asymptotics for the finite-time ruin probability of a dependent risk model with a constant interest rate. Methodol. Comput. Appl. Probab. 15, 109-124 (2003)

11. Ng, KW, Tang, Q, Yan, J, Yang, H: Precise large deviations for the prospective-loss process. J. Appl. Probab. 40, 391-400 (2003)

10.1186/1029-242X-2014-140

Cite this article as: Bai et al.: Large deviations for randomly weighted sums with dominantly varying tails and widely orthant dependent structure. Journal of Inequalities and Applications 2014, 2014:140 